\title{
Examining the role of socioeconomic deprivation in ethnic differences in sexually transmitted infection diagnosis rates in England: evidence from surveillance data
}

\author{
M. FUREGATO ${ }^{*} \dagger$, Y. $\mathrm{CHEN}^{2} \dagger$, H. MOHAMMED ${ }^{1}$, C. H. MERCER ${ }^{2}$, \\ E. J. SAVAGE ${ }^{1}$ AND G. HUGHES ${ }^{1}$ \\ ${ }^{1}$ National Infection Service, Public Health England, London, UK \\ ${ }^{2}$ Centre for Sexual Health and HIV Research, University College London, London, UK
}

Received 22 January 2016; Final revision 14 June 2016; Accepted 10 July 2016; first published online 11 August 2016

\section{SUMMARY}

Differences by ethnic group in STI diagnosis rates have long been recognized in England. We investigated whether these may be explained by ethnic disparities in socioeconomic deprivation (SED). Data on all diagnoses made in sexual health clinics in England in 2013 were obtained from the mandatory STI surveillance system. Poisson regression was used to calculate incidence rate ratios (IRRs) of STIs, by ethnicity, with and without adjustment for index of multiple deprivation (IMD) a measure of area-level deprivation. Unadjusted IRRs (95\% confidence intervals) were highest for gonorrhoea [8.18 (7.77-8.61) and 5.76 (5.28-6.29)] and genital herpes [4.24 (3.99-4.51) and $3.58(3 \cdot 23-3 \cdot 98)$ ] for people of black Caribbean and non-Caribbean/non-African black ethnicity

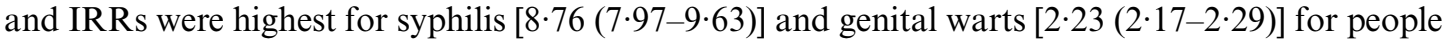
of non-British/non-Irish white ethnicity compared to white British ethnicity. After adjustment for IMD, IRRs for gonorrhoea [5.76 (5.47-6.07)] and genital herpes [3.73 (3.50-3.97)] declined but remained highest for black Caribbeans and IRRs for syphilis [7.35 (6.68-8.09)] and genital warts [2.10 (2.04-2.16)] declined but remained highest for non-British/non-Irish white compared to white British. In England, ethnic disparities in STI diagnosis rates are partially explained by SED, but behavioural and contextual factors likely contribute. Clinic and community-based interventions should involve social peer networks to ensure they are targeted and culturally sensitive.

Key words: ethnicity, IMD, socioeconomic deprivation, STI.

\section{INTRODUCTION}

Ethnic disparities in the rates of sexually transmitted infection (STI) diagnoses have been reported in many developed countries and are a major source of health inequality worldwide $[1,2]$. In the UK, disproportionately high STI and HIV rates are reported in

\footnotetext{
* Author for correspondence: Ms. M. Furegato, National Infection Service, Public Health England, 61 Colindale Avenue, London NW9 5EQ, UK.

(Email: Martina.Furegato@phe.gov.uk)

$\dagger$ These authors served as joint first authors.
}

people of black Caribbean and black African ethnicity [3, 4]. In England, among sexual health clinic attendees, the highest diagnosis rates of gonorrhoea, genital herpes, genital warts and syphilis are found in black ethnic groups [5-7].

Socioeconomic deprivation (SED) is one of the major determinants of poor health [8], and it is also frequently implicated as a contributor to the disparate health observed in racial and ethnic minorities [9]. SED refers to the range of socioeconomic circumstances, such as income, education and occupation, by which individuals are hierarchically stratified in 
society [8], and can be expressed as poor access to healthcare [10], poor education, social segregation $[11,12]$ and poor housing [13]. A link between decreasing socioeconomic status and increased risk of a multitude of diseases, including infectious diseases such as STIs, has been already established [14, 15].

Especially for people of lower socioeconomic status, engaging in high-risk behaviour could be linked to poor self-esteem, perceived limitations of life choices and limited control over what happens to their health [16]. Behavioural risk factors are themselves linked to the social gradient by levels of risk associated with the social and structural environment [17]. A living environment with low social capital places an individual at increased risk of exposure to infections associated with behavioural risk [18]. In addition, racial disparities in sexual and in general health typically reflect environmental and social differences between racial groups $[1,19]$.

A previous analysis investigated the association between SED and ethnicity in terms of STI risk [20]. This analysis highlighted that the STI diagnosis rates in black ethnic communities remained significantly higher than those of other ethnic groups after adjustment for SED. However, the analysis was based on patients' lower-tier Local Authority (LA) of residence, large administrative units of local government, of which there are 326 in England. In this paper, we refine and update these analyses using a much smaller geographical unit, the Lower Super Output Area (LSOA), 32482 census output areas with an average population of 1620 persons [21], to investigate the association between ethnicity, STI diagnosis rates and SED in England.

\section{METHODS}

Data from all 215 sexual health clinics in England were obtained from the Genitourinary Medicine Clinic Activity Dataset version 2 (GUMCADv2), the mandatory surveillance system for all STI diagnoses and services in England [22]. All sexual health clinic attendances from 1 January 2013 to 31 December 2013, inclusive, were considered in the analysis. The diagnosis rates per 100000 population of gonorrhoea; primary, secondary and early latent syphilis; genital warts (first episode); and genital herpes (first episode) were derived.

SED was measured using the index of multiple deprivation (IMD) a measure of area-level deprivation for each LSOA. The IMD score [23], is constructed for each of 32482 defined LSOAs in England by combining scores derived largely from routine administrative data for the following seven domains (weighted for importance): income (22.5\%), employment $(22 \cdot 5 \%)$, health and disability $(13 \cdot 5 \%)$, education, skills and training $(13.5 \%)$, barriers to housing and services $(9 \cdot 3 \%)$, crime $(9 \cdot 3 \%)$, living environment $(9 \cdot 3 \%)$ [24].

Each LSOA was ranked according to the IMD score, and then assigned to quintiles. Denominators used to derive crude incidence rates of STI diagnoses were obtained from the 2011 Census [25]. Poisson regression was used to calculate unadjusted and IMD-adjusted incidence rate ratios (IRRs) for each STI by ethnic group. As census data only provide limited demographic breakdowns by LSOA, demographic factors other than ethnicity could not be considered in the Poisson regression model.

A sensitivity analysis to examine the relationship between ethnicity, deprivation and other demographic factors was performed using binary logistic regression to derive odds ratios (ORs) for the diagnosis of each STI among sexual health clinic patients, with and without adjustment for IMD, age and gender/sexual orientation. Gender and sexual orientation were combined as a single variable consisting of the following categories: men who have sex with men, heterosexual men and women $(<1 \%$ of women were lesbian, so this was not considered as a category due to small cell sizes for analysis).

All analyses were performed using Stata v. 13.1 (StataCorp LP, USA) [26], and $P$ values $<5 \%$ were considered statistically significant.

\section{Ethical standards}

Ethics committee approval is not required, as the analyses are based on surveillance data held by Public Health England. These datasets have approval for analyses for public health purposes.

\section{RESULTS}

In England, there was little variation in the distribution of white British people by IMD quintile of their LSOA of residence: $22 \%$ of white British people lived in the least deprived areas and $17 \%$ lived in the most deprived areas (Fig. 1). This contrasted with other ethnic groups. For example, $47 \%$ of black British people lived in the most deprived areas, while only $4 \%$ lived in the least deprived areas (Fig. 1). 


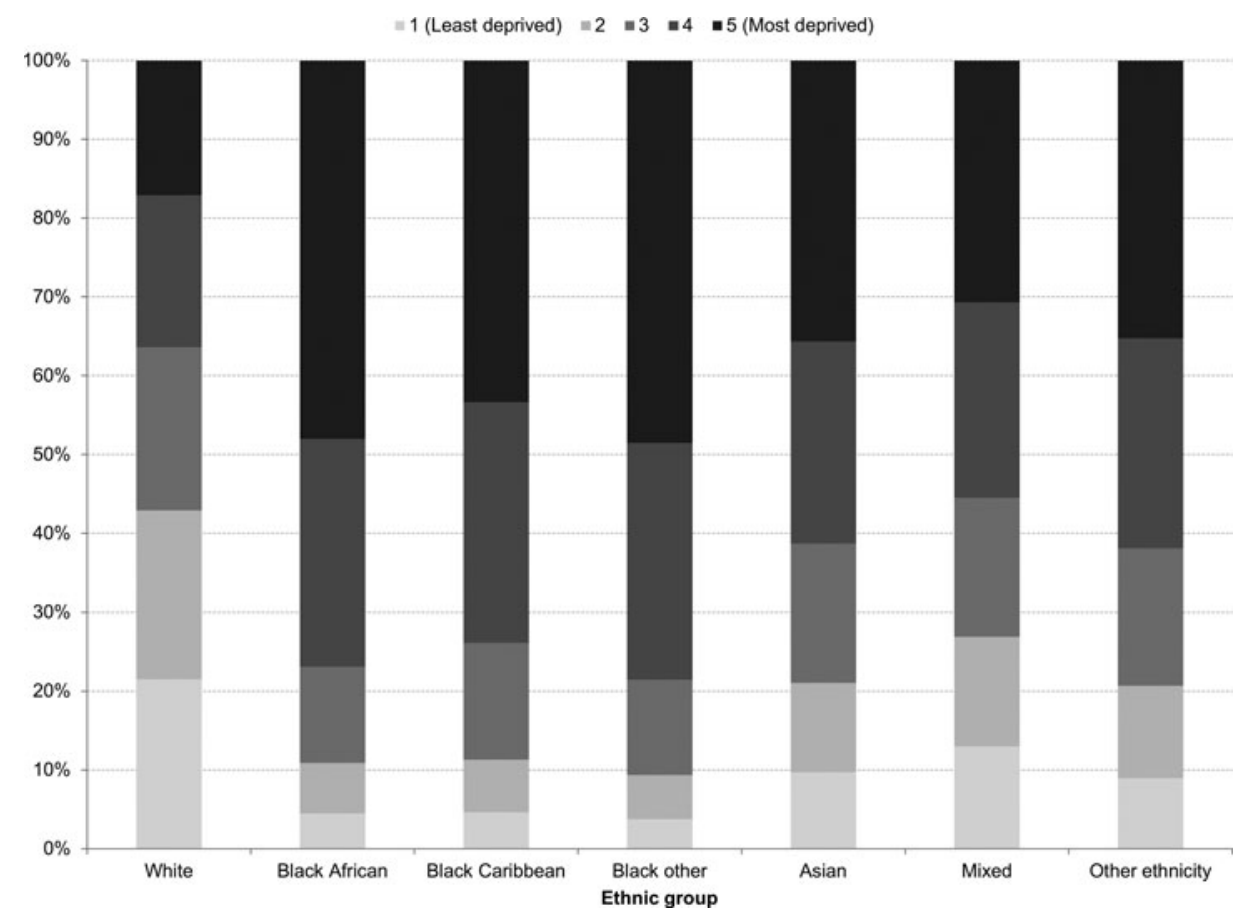

Fig. 1. Ethnic variations in the distribution of the index of multiple deprivation in England, 2011.

In 2013, data from 2539572 sexual health clinic attendances were submitted to GUMCADv2 and the proportion of attendances reported with known ethnicity was $99 \cdot 7 \%$.

Most $(65 \cdot 3 \%)$ of the attendances were by patients of white British ethnicity, followed by those of nonBritish/non-Irish white ethnicity (10.7\%) and black African ethnicity $(6 \cdot 4 \%)$. The proportions of attendances by people of black Caribbean and nonCaribbean/non-African black ethnicity were $3.9 \%$ and $2 \cdot 0 \%$, respectively.

Black Caribbeans had the highest crude rates per 100000 population for gonorrhoea (285.7) and genital herpes (190.0), while people of non-British/non-Irish white ethnicity had the highest rates of genital warts (228.4) and syphilis (25.8). The crude rates in those of white British ethnicity were 34.9 for gonorrhoea, 51.4 for genital herpes, 123.6 for genital warts and 3.6 for syphilis (Fig. 2).

Unadjusted IRRs (95\% confidence intervals) from the Poisson regression were highest for gonorrhoea [8.18 (7.77-8.61) and 5.76 (5.28-6.29)] and genital herpes [4.24 (3.99-4.51) and 3.58 (3.23-3.98)] for people of black Caribbean and non-Caribbean/ non-African black ethnicity, respectively, compared to those of white British ethnicity (Table 1). Unadjusted IRRs were highest for people of
non-British/non-Irish white ethnicity for syphilis [8.76 (7.97-9.63)] and genital warts [2.23 (2.172.29)], respectively, compared to those of white British ethnicity (Table 1).

After adjustment for IMD, IRRs for gonorrhoea [5.76 (5.47-6.07)] and genital herpes [3.73 (3.503.97)] declined but remained highest for black Caribbeans compared to those of white British ethnicity (Table 2). IRRs for syphilis [7.35 (6.68-8.09)] and genital warts [2.10 (2.04-2.16)] also declined but remained highest for non-British/non-Irish white ethnicity compared to those of white British ethnicity (Table 2).

People of Indian and Pakistani ethnicity had consistently lower IRRs (both unadjusted and adjusted) for gonorrhoea, genital warts and genital herpes compared to white British people (Tables 1 and 2).

According to the sensitivity analysis (Table 3), the ORs for gonorrhoea [1.91 (1.82-2.02) and 1.61 $(1 \cdot 48-1 \cdot 76)]$ were highest for black Caribbean and people of non-Caribbean/non-African black ethnicity, respectively, compared to white British ethnic groups. In contrast, the ORs for syphilis [1.64 (1.21-2.21)] were highest for those of non-Caribbean/non-African black ethnicity. The ORs for genital warts and genital herpes were highest in those of white British ethnicity. 

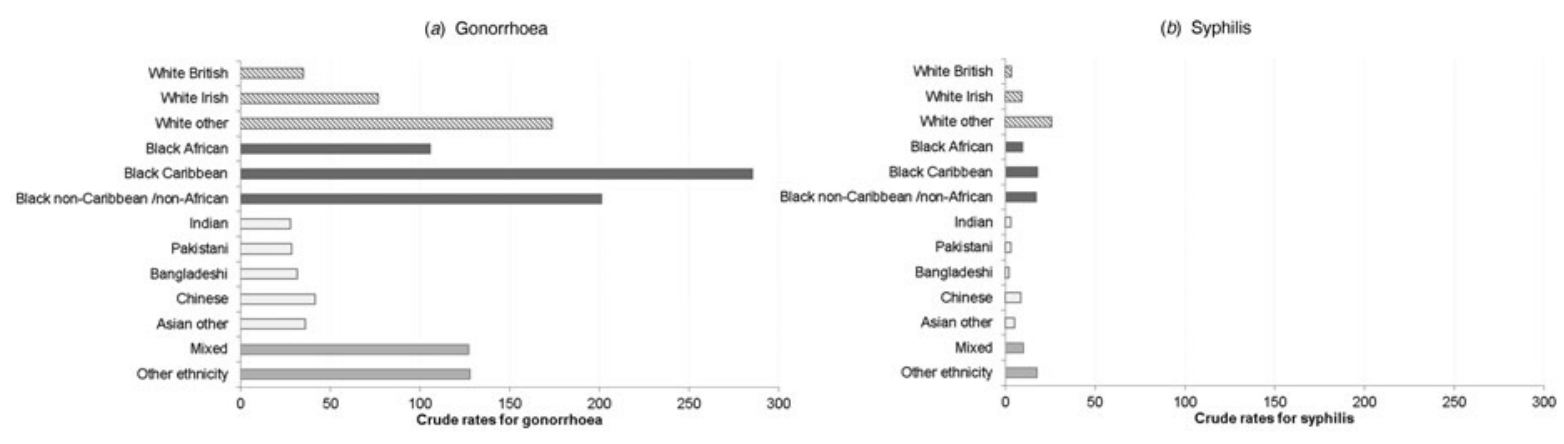

(c) Genital herpes

(d) Genital warts
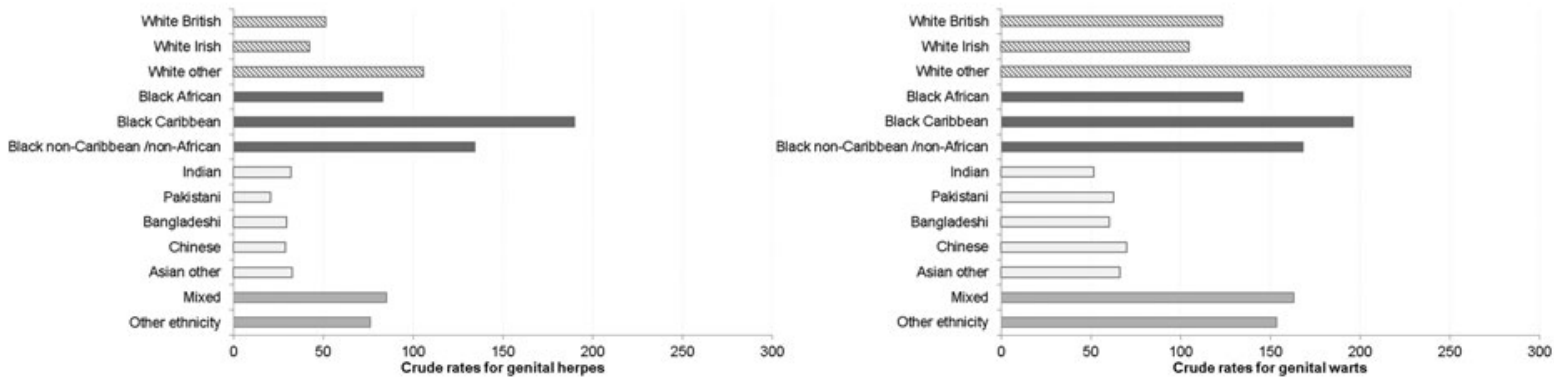

Fig. 2. Crude rates for $(a)$ gonorrhea, $(b)$ syphilis, $(c)$ genital herpes and $(d)$ genital warts by ethnic group, England, 2013.

\section{DISCUSSION}

After controlling for deprivation, the strength of association between ethnicity and STI diagnosis was reduced, most notably for gonorrhoea in those of black Caribbean and non-Caribbean/non-African black ethnicity, suggesting that socioeconomic status and poverty might be important correlates of racial disparities in health. However, variation by ethnicity persisted. After additional adjustment for sexual orientation and age, persons of black Caribbean ethnicity remained the ethnic group with the greatest odds of gonorrhoea diagnoses.

Compared to the previous analysis performed which included larger geographical units [20], the refined version presented here allowed us to investigate the association between STI diagnoses, ethnicity and SED, at a very small local level (LSOA). Confounding variables have been included as much as possible given population data availability at the local level. In this respect, one limitation of this study relates to the use of IMD. This is a measure of residential area-level and not individual deprivation, and thus is subject to ecological fallacy. Furthermore, although IMD is composed of many closely related domains, chosen to reflect varying forms of disadvantage, the indicator provides no insight into which specific factors are associated with the pathway between exposure and infection.
Although we were able to adjust for SED, residual confounding suggests that there are unknown-and possibly unmeasurable - predictors for some infections. As such, we performed a sensitivity analysis to adjust for multiple demographic factors in addition to IMD, and similar results for the bacterial STIs with respect to IMD, ethnicity and STI diagnoses were observed. Other confounders such as risky sexual behaviour and drug use could not be included in this study as these characteristics are not currently collected by GUMCADv2. However, the enhancement of GUMCADv2 [27] to include behavioural information is currently being piloted from a subset of STI services, and future studies may be able to address this study's limitation.

While chlamydia is the most common STI to be diagnosed in England [28], it was not considered in this analysis because $48 \%$ of diagnoses are made in different settings such as sexual and reproductive health clinics, general practice, Young people's services. In addition, data are captured from another surveillance system [29], which has poor data quality on ethnicity which could potentially bias the results. In contrast, one of this study's most important strengths is that we used national surveillance data which benefits from $100 \%$ reporting compliance and high data completion (each variable collected has at least $90 \%$ completion), resulting in a dataset with over 2 million 
Table 1. Unadjusted incidence rate ratios (IRRs) for gonorrhoea, syphilis*, genital herpes and genital warts by ethnic group, England, 2013

\begin{tabular}{|c|c|c|c|c|c|c|c|c|c|c|c|c|c|c|c|c|}
\hline \multirow[b]{2}{*}{ Ethnic group } & \multicolumn{4}{|c|}{ Gonorrhoea $(N=25238)$} & \multicolumn{4}{|c|}{ Syphilis $(N=2710)$} & \multicolumn{4}{|c|}{ Genital herpes $(N=28465)$} & \multicolumn{4}{|c|}{ Genital warts $(N=64372)$} \\
\hline & $\%$ & IRR & $(95 \% \mathrm{CI})$ & value & $\%$ & IRR & $(95 \% \mathrm{CI})$ & value & $\%$ & IRR & $(95 \% \mathrm{CI})$ & $P$ value & $\%$ & IRR & $(95 \% \mathrm{CI})$ & $P$ val \\
\hline $\mathrm{v}$ & 7 & 1 & & & ? & 1 & & & 7 & 1 & & & & 1 & & \\
\hline White Irish & & $2 \cdot 19$ & $(1.98$ & 1 & 1 & $6 \cdot 78$ & $(5 \cdot 06$ & 1 & & $2 \cdot 15$ & 6) & 1 & $0 \cdot 8$ & $2 \cdot 21$ & & 001 \\
\hline White other & $16 \cdot 2$ & $4 \cdot 97$ & $(4 \cdot 80-5 \cdot 15)$ & 01 & $22 \cdot 4$ & $8 \cdot 76$ & $(7 \cdot 97-9 \cdot 63)$ & $\cdot 001$ & $8 \cdot 7$ & $2 \cdot 48$ & $(2 \cdot 38-2 \cdot 59)$ & $<0.001$ & 0.0 & $2 \cdot 23$ & & $0 \cdot 001$ \\
\hline Mixed & 5.7 & $3 \cdot 64$ & $(3 \cdot 45-3 \cdot 84)$ & $\cdot 001$ & $4 \cdot 2$ & $3 \cdot 97$ & $(3 \cdot 28-4 \cdot 80)$ & $<0.001$ & $3 \cdot 4$ & $2 \cdot 32$ & $(2 \cdot 17-2 \cdot 47)$ & $<0.001$ & $2 \cdot 9$ & $1 \cdot 84$ & & $<0 \cdot 001$ \\
\hline Indian & & $0 \cdot 8$ & $(0.72-0.88)$ & $<0 \cdot 0$ & $1 \cdot 6$ & $1 \cdot 21$ & $(0.85$ & 0. & 1 . & $0 \cdot 83$ & 1) & $<0$ & $1 \cdot 1$ & $0 \cdot 56$ & & $<0 \cdot 001$ \\
\hline Pakis & & & & & & & & & & & & & & & & $<0 \cdot 001$ \\
\hline Bangladeshi & $0 \cdot 5$ & $0 \cdot 91$ & $(0 \cdot 7$ & 78 & 0 & $0 \cdot 97$ & $(0$. & 4 & $0 \cdot 4$ & $0 \cdot 91$ & 08) & 0 & $0 \cdot 4$ & $0 \cdot 77$ & $(0$ & $<0.001$ \\
\hline Chinese & 0.6 & $1 \cdot 19$ & $(1 \cdot 01-1 \cdot 41)$ & 38 & & $6 \cdot 2$ & $(4 \cdot 29-8 \cdot 95)$ & $<0 \cdot 001$ & $0 \cdot 3$ & $1 \cdot 45$ & $(1 \cdot 19-1 \cdot 77)$ & $<0 \cdot 001$ & $0 \cdot 4$ & $1 \cdot 46$ & $(1 \cdot 28-1 \cdot 65)$ & $<0 \cdot 001$ \\
\hline Asian other & $1 \cdot 1$ & $1 \cdot 04$ & $(0 \cdot 92-1 \cdot 17)$ & & & & & & & & & & & & & 0.037 \\
\hline Black African & $3 \cdot 9$ & & & & $3 \cdot 4$ & & & & & & & & & & & $<0 \cdot 001$ \\
\hline Black Caribbean & $6 \cdot 4$ & $8 \cdot 18$ & $(7 \cdot 77-8 \cdot 61)$ & $<0 \cdot 001$ & $3 \cdot 8$ & $5 \cdot 83$ & $(4 \cdot 77-7 \cdot 13)$ & $<0.001$ & $3 \cdot 8$ & $4 \cdot 24$ & $(3.99-4.51)$ & $<0.001$ & $1 \cdot 7$ & $1 \cdot 82$ & $(1 \cdot 72-1 \cdot 94)$ & $<0 \cdot 001$ \\
\hline Black other ${ }^{\dagger}$ & $2 \cdot 2$ & $5 \cdot 76$ & $(5 \cdot 28-6 \cdot 29)$ & $<0 \cdot 001$ & $1 \cdot 7$ & $6 \cdot 75$ & $(5 \cdot 03-9 \cdot 05)$ & $<0.001$ & $1 \cdot 3$ & $3 \cdot 58$ & $(3 \cdot 23-3 \cdot 98)$ & $<0.001$ & $0 \cdot 7$ & $1 \cdot 87$ & $(1 \cdot 70-2 \cdot 04)$ & $<0 \cdot 001$ \\
\hline Other ethnicity & $2 \cdot 6$ & $3 \cdot 67$ & $(3 \cdot 39-3 \cdot 97)$ & $<0 \cdot 001$ & $3 \cdot 4$ & $7 \cdot 22$ & $(5 \cdot 84-8 \cdot 92)$ & $<0.001$ & $1 \cdot 4$ & $2 \cdot 13$ & $(1.93-2 \cdot 36)$ & $<0.001$ & $1 \cdot 2$ & $1 \cdot 79$ & $(1.67-1.93)$ & $<0 \cdot 001$ \\
\hline
\end{tabular}

CI, Confidence interval.

* Syphilis is defined as primary, secondary and early latent syphilis.

$\dagger$ Black other: non-Caribbean/non-African black ethnicity. 
Table 2. Incidence rate ratios (IRRs) for gonorrhoea, syphilis*, genital herpes and genital warts by ethnic group adjusted for index of multiple deprivation (IMD), England-2013

\begin{tabular}{|c|c|c|c|c|c|c|c|c|c|c|c|c|c|c|c|c|}
\hline & \multicolumn{4}{|c|}{ Gonorrhoea $(N=25238)$} & \multicolumn{4}{|c|}{ Syphilis $(N=2710)$} & \multicolumn{4}{|c|}{ Genital herpes $(N=28465)$} & \multicolumn{4}{|c|}{ Genital warts $(N=64372)$} \\
\hline & $\%$ & IRR & $(95 \% \mathrm{CI})$ & $P$ value & $\%$ & IRR & $(95 \% \mathrm{CI})$ & $P$ value & $\%$ & IRR & $(95 \% \mathrm{CI})$ & $P$ value & $\%$ & IRR & $(95 \% \mathrm{CI})$ & $P$ value \\
\hline \multicolumn{17}{|l|}{ Ethnic group } \\
\hline White British & $56 \cdot 7$ & 1 & & & $53 \cdot 8$ & 1 & & & $74 \cdot 0$ & 1 & & & $78 \cdot 6$ & 1 & & \\
\hline White Irish & $1 \cdot 5$ & $2 \cdot 01$ & $(1 \cdot 82-2 \cdot 23)$ & $<0.001$ & $1 \cdot 7$ & $5 \cdot 63$ & $(4 \cdot 19-7 \cdot 55)$ & $<0.001$ & $0 \cdot 7$ & $2 \cdot 02$ & $(1 \cdot 76-2 \cdot 31)$ & $<0 \cdot 001$ & $0 \cdot 8$ & $2 \cdot 08$ & $(1 \cdot 91-2 \cdot 27)$ & $=0 \cdot 001$ \\
\hline White other & $16 \cdot 2$ & $4 \cdot 26$ & $(4 \cdot 11-4 \cdot 41)$ & $<0.001$ & $22 \cdot 4$ & $7 \cdot 35$ & $(6 \cdot 68-8 \cdot 09)$ & $<0.001$ & $8 \cdot 7$ & $2 \cdot 34$ & $(2 \cdot 24-2 \cdot 44)$ & $<0 \cdot 001$ & $8 \cdot 3$ & $2 \cdot 10$ & $(2 \cdot 04-2 \cdot 16)$ & $<0 \cdot 001$ \\
\hline Mixed & $5 \cdot 7$ & $2 \cdot 99$ & $(2 \cdot 83-3 \cdot 15)$ & $<0.001$ & $4 \cdot 2$ & $3 \cdot 11$ & $(2 \cdot 57-3 \cdot 77)$ & $<0.001$ & $3 \cdot 4$ & $2 \cdot 12$ & $(1 \cdot 99-2 \cdot 26)$ & $<0 \cdot 001$ & $2 \cdot 9$ & $1 \cdot 69$ & $(1 \cdot 61-1 \cdot 77)$ & $<0 \cdot 001$ \\
\hline Indian & $1 \cdot 5$ & $0 \cdot 68$ & $(0 \cdot 61-0 \cdot 75)$ & $<0.001$ & $1 \cdot 6$ & $1 \cdot 00$ & $(0 \cdot 74-1 \cdot 36)$ & 0.987 & $1 \cdot 5$ & $0 \cdot 77$ & $(0 \cdot 70-0 \cdot 85)$ & $<0 \cdot 001$ & $1 \cdot 1$ & $0 \cdot 52$ & $(0 \cdot 49-0 \cdot 56)$ & $<0 \cdot 001$ \\
\hline Pakistani & $1 \cdot 2$ & 0.56 & $(0 \cdot 50-0.63)$ & $<0.001$ & $1 \cdot 2$ & $0 \cdot 76$ & $(0 \cdot 53-1 \cdot 07)$ & $0 \cdot 115$ & $0 \cdot 8$ & $0 \cdot 44$ & $(0 \cdot 38-0 \cdot 50)$ & $<0 \cdot 001$ & $1 \cdot 1$ & $0 \cdot 55$ & $(0 \cdot 51-0 \cdot 60)$ & $<0 \cdot 001$ \\
\hline Bangladeshi & $0 \cdot 5$ & $0 \cdot 60$ & $(0 \cdot 51-0 \cdot 72)$ & $<0.001$ & $0 \cdot 3$ & $0 \cdot 64$ & $(0 \cdot 33-1 \cdot 23)$ & $0 \cdot 182$ & $0 \cdot 4$ & $0 \cdot 77$ & $(0.65-0.92)$ & 0.005 & $0 \cdot 4$ & $0 \cdot 65$ & $(0 \cdot 58-0.74)$ & $<0 \cdot 001$ \\
\hline Chinese & $0 \cdot 6$ & $1 \cdot 06$ & $(0 \cdot 90-1 \cdot 25)$ & 0.492 & $1 \cdot 1$ & $5 \cdot 17$ & $(3 \cdot 58-7 \cdot 48)$ & $<0.001$ & $0 \cdot 3$ & $1 \cdot 36$ & $(1 \cdot 11-1 \cdot 66)$ & $<0 \cdot 001$ & $0 \cdot 4$ & $1 \cdot 37$ & $(1 \cdot 20-1 \cdot 55)$ & $<0 \cdot 001$ \\
\hline Asian other & $1 \cdot 1$ & $0 \cdot 86$ & $(0 \cdot 76-0 \cdot 96)$ & $0 \cdot 01$ & $1 \cdot 5$ & 1.95 & $(1 \cdot 43-2 \cdot 67)$ & $<0.001$ & $0 \cdot 9$ & $1 \cdot 00$ & $(0 \cdot 88-1 \cdot 13)$ & 0.996 & $0 \cdot 8$ & $0 \cdot 84$ & $(0.77-0.92)$ & $<0.001$ \\
\hline Black African & $3 \cdot 9$ & $2 \cdot 09$ & $(1 \cdot 96-2 \cdot 24)$ & $<0.001$ & $3 \cdot 4$ & $\mathbf{2} \cdot 17$ & $(1 \cdot 75-2 \cdot 68)$ & $<0.001$ & $2 \cdot 7$ & $1 \cdot 56$ & $(1 \cdot 45-1 \cdot 68)$ & $<0 \cdot 001$ & $1 \cdot 9$ & $1 \cdot 06$ & $(1 \cdot 00-1 \cdot 12)$ & 0.059 \\
\hline Black Caribbean & $6 \cdot 4$ & $5 \cdot 76$ & $(5 \cdot 47-6 \cdot 07)$ & $<0 \cdot 001$ & $3 \cdot 8$ & $4 \cdot 11$ & $(3 \cdot 35-5 \cdot 03)$ & $<0.001$ & $3 \cdot 8$ & $3 \cdot 73$ & $(3 \cdot 50-3 \cdot 97)$ & $<0 \cdot 001$ & $1 \cdot 7$ & $1 \cdot 60$ & $(1 \cdot 51-1 \cdot 70)$ & $<0 \cdot 001$ \\
\hline Black other $^{\dagger}$ & $2 \cdot 2$ & 3.93 & $(3 \cdot 60-4 \cdot 29)$ & $<0.001$ & $1 \cdot 7$ & $4 \cdot 58$ & $(3 \cdot 41-6 \cdot 15)$ & $<0.001$ & $1 \cdot 3$ & $3 \cdot 10$ & $(2 \cdot 79-3 \cdot 45)$ & $<0 \cdot 001$ & $0 \cdot 7$ & $1 \cdot 61$ & $(1 \cdot 46-1 \cdot 77)$ & $<0 \cdot 001$ \\
\hline Other ethnicity & $2 \cdot 6$ & $2 \cdot 84$ & $(2 \cdot 62-3 \cdot 07)$ & $<0.001$ & $3 \cdot 4$ & $5 \cdot 42$ & $(4 \cdot 38-6 \cdot 71)$ & $<0.001$ & $1 \cdot 4$ & 1.92 & $(1 \cdot 74-2 \cdot 13)$ & $<0.001$ & $1 \cdot 2$ & $1 \cdot 62$ & $(1 \cdot 51-1 \cdot 74)$ & $<0 \cdot 001$ \\
\hline \multicolumn{17}{|l|}{ IMD quintile } \\
\hline 1 (least deprived) & $7 \cdot 8$ & 1 & & & $7 \cdot 3$ & 1 & & & $14 \cdot 6$ & 1 & & & $15 \cdot 8$ & 1 & & \\
\hline 2 & $10 \cdot 3$ & $1 \cdot 29$ & $(1 \cdot 22-1 \cdot 37)$ & $<0.001$ & $10 \cdot 8$ & $1 \cdot 38$ & $(1 \cdot 15-1 \cdot 65)$ & & $16 \cdot 7$ & $1 \cdot 11$ & $(1 \cdot 06-1 \cdot 15)$ & $<0 \cdot 001$ & $17 \cdot 4$ & $1 \cdot 08$ & $\cdot 11)$ & $<0 \cdot 001$ \\
\hline 3 & $15 \cdot 6$ & $1 \cdot 82$ & $(1 \cdot 73-1.93)$ & $<0.001$ & $16 \cdot 5$ & 1.91 & $(1 \cdot 61-2 \cdot 26)$ & $<0.001$ & $19 \cdot 6$ & $1 \cdot 25$ & $(1 \cdot 20-1 \cdot 30)$ & $<0 \cdot 001$ & $19 \cdot 1$ & $1 \cdot 16$ & $(1 \cdot 13-1 \cdot 19)$ & $<0 \cdot 001$ \\
\hline 4 & $30 \cdot 2$ & $3 \cdot 18$ & $(3 \cdot 03-3 \cdot 34)$ & $<0 \cdot 001$ & $31 \cdot 8$ & $3 \cdot 17$ & $(2 \cdot 71-3 \cdot 71)$ & $<0.001$ & $23 \cdot 8$ & $1 \cdot 42$ & $(1 \cdot 37-1 \cdot 48)$ & $<0 \cdot 001$ & $23 \cdot 6$ & $1 \cdot 39$ & $(1 \cdot 36-1 \cdot 43)$ & $<0 \cdot 001$ \\
\hline 5 (most deprived) & $36 \cdot 1$ & $3 \cdot 66$ & $(3 \cdot 48-3 \cdot 85)$ & $<0.001$ & $33 \cdot 6$ & $3 \cdot 24$ & $(2 \cdot 76-3 \cdot 79)$ & $<0.001$ & $25 \cdot 3$ & $1 \cdot 48$ & $(1 \cdot 42-1 \cdot 54)$ & $<0.001$ & $24 \cdot 1$ & $1 \cdot 44$ & $(1 \cdot 40-1 \cdot 48)$ & $<0 \cdot 001$ \\
\hline
\end{tabular}

CI, Confidence interval.

* Syphilis is defined as primary, secondary and early latent syphilis.

$\dagger$ Black other: non-Caribbean/non-African black ethnicity. 
Table 3. Adjusted odds ratios (aORs) for gonorrhoea, syphilis*, genital herpes and genital warts diagnoses by ethnic group, England, 2013

\begin{tabular}{|c|c|c|c|c|c|c|c|c|c|c|c|c|c|c|c|c|}
\hline & \multicolumn{4}{|c|}{ Gonorrhoea $(N=27115)$} & \multicolumn{4}{|c|}{ Syphilis $(N=2953)$} & \multicolumn{4}{|c|}{ Genital herpes $(N=29986)$} & \multicolumn{4}{|c|}{ Genital warts $(N=67850)$} \\
\hline & $\%$ & $\mathrm{aOR}$ & $(95 \% \mathrm{CI})$ & $P$ value & $\%$ & $\mathrm{aOR}$ & $(95 \% \mathrm{CI})$ & $P$ value & $\%$ & $\mathrm{aOR}$ & $(95 \% \mathrm{CI})$ & $P$ value & $\%$ & $\mathrm{aOR}$ & $(95 \% \mathrm{CI})$ & $P$ value \\
\hline \multicolumn{17}{|l|}{ Ethnic group } \\
\hline White British & $56 \cdot 6$ & 1 & & & $54 \cdot 3$ & 1 & & & $78 \cdot 3$ & 1 & & & $73 \cdot 5$ & 1 & & \\
\hline White Irish & $1 \cdot 5$ & $\mathbf{1 \cdot 2 7}$ & $(1 \cdot 14-1 \cdot 40)$ & $<0 \cdot 001$ & $1 \cdot 7$ & $1 \cdot 13$ & $(0 \cdot 85-1 \cdot 50)$ & $0 \cdot 390$ & $0 \cdot 8$ & $0 \cdot 77$ & $(0 \cdot 67-0 \cdot 87)$ & $<0 \cdot 001$ & $0 \cdot 8$ & $0 \cdot 84$ & $(0 \cdot 77-0 \cdot 92)$ & $<0 \cdot 001$ \\
\hline White other & $16 \cdot 3$ & $1 \cdot 25$ & $(1 \cdot 21-1 \cdot 30)$ & $<0 \cdot 001$ & $22 \cdot 0$ & $1 \cdot 46$ & $(1 \cdot 33-1 \cdot 61)$ & $<0 \cdot 001$ & $8 \cdot 5$ & $0 \cdot 78$ & $(0 \cdot 75-0 \cdot 81)$ & $<0 \cdot 001$ & $8 \cdot 9$ & $0 \cdot 78$ & $(0 \cdot 76-0 \cdot 80)$ & $<0 \cdot 001$ \\
\hline Mixed & $5 \cdot 7$ & $1 \cdot 52$ & $(1 \cdot 44-1 \cdot 60)$ & $<0 \cdot 001$ & $4 \cdot 1$ & $1 \cdot 40$ & $(1 \cdot 16-1 \cdot 69)$ & $<0.001$ & $2 \cdot 9$ & 0.74 & $(0.70-0.79)$ & $<0.001$ & $3 \cdot 4$ & 0.59 & $(0.57-0.62)$ & $<0 \cdot 001$ \\
\hline Indian & $1 \cdot 4$ & $0 \cdot 97$ & $(0 \cdot 88-1 \cdot 08)$ & $0 \cdot 610$ & $1 \cdot 7$ & $1 \cdot 21$ & $(0 \cdot 91-1 \cdot 62)$ & $0 \cdot 180$ & $1 \cdot 1$ & $0 \cdot 82$ & $(0 \cdot 75-0.91)$ & $<0 \cdot 001$ & $1 \cdot 5$ & $0 \cdot 57$ & $(0.53-0.61)$ & $<0 \cdot 001$ \\
\hline Pakistani & $1 \cdot 2$ & $1 \cdot 08$ & $(0 \cdot 97-1 \cdot 22)$ & $0 \cdot 170$ & $1 \cdot 1$ & $1 \cdot 25$ & $(0 \cdot 88-1 \cdot 79)$ & $0 \cdot 220$ & $1 \cdot 1$ & 0.66 & $(0.58-0.75)$ & $<0 \cdot 001$ & $0 \cdot 8$ & $0 \cdot 75$ & $(0 \cdot 70-0.82)$ & $<0 \cdot 001$ \\
\hline Bangladeshi & $0 \cdot 5$ & $1 \cdot 15$ & $(0 \cdot 97-1 \cdot 36)$ & $0 \cdot 110$ & $0 \cdot 3$ & $1 \cdot 01$ & $(0 \cdot 52-1 \cdot 95)$ & 0.970 & $0 \cdot 4$ & $0 \cdot 76$ & $(0.63-0.90)$ & $<0 \cdot 001$ & $0 \cdot 4$ & 0.62 & $(0 \cdot 55-0 \cdot 70)$ & $<0 \cdot 001$ \\
\hline Chinese & 0.6 & 0.73 & $(0.63-0.85)$ & $<0.001$ & $1 \cdot 2$ & $1 \cdot 40$ & $(1 \cdot 01-1 \cdot 95)$ & $0 \cdot 050$ & $0 \cdot 4$ & 0.59 & $(0 \cdot 49-0 \cdot 70)$ & $<0.001$ & $0 \cdot 4$ & $0 \cdot 60$ & $(0.53-0.68)$ & $<0 \cdot 001$ \\
\hline Asian other & $1 \cdot 1$ & $0 \cdot 98$ & $(0 \cdot 87-1 \cdot 10)$ & $0 \cdot 710$ & $1 \cdot 5$ & $1 \cdot 27$ & $(0.94-1 \cdot 73)$ & $0 \cdot 120$ & $0 \cdot 8$ & $0 \cdot 71$ & $(0 \cdot 63-0.80)$ & $<0 \cdot 001$ & $0 \cdot 9$ & $0 \cdot 63$ & $(0.58-0.68)$ & $<0 \cdot 001$ \\
\hline Black African & $3 \cdot 9$ & $1 \cdot 04$ & $(0 \cdot 98-1 \cdot 11)$ & $0 \cdot 210$ & $3 \cdot 3$ & $1 \cdot 22$ & $(0 \cdot 98-1 \cdot 51)$ & $0 \cdot 080$ & $2 \cdot 0$ & $0 \cdot 38$ & $(0 \cdot 35-0 \cdot 41)$ & $<0 \cdot 001$ & $2 \cdot 8$ & $0 \cdot 31$ & $(0 \cdot 29-0.33)$ & $<0 \cdot 001$ \\
\hline Black Caribbean & $6 \cdot 3$ & 1.91 & $(1 \cdot 82-2 \cdot 02)$ & $<0 \cdot 001$ & $3 \cdot 6$ & $1 \cdot 38$ & $(1 \cdot 13-1 \cdot 70)$ & $<0 \cdot 001$ & $1 \cdot 7$ & $0 \cdot 75$ & $(0 \cdot 70-0 \cdot 80)$ & $<0 \cdot 001$ & $3 \cdot 8$ & $0 \cdot 34$ & $(0 \cdot 32-0 \cdot 36)$ & $<0 \cdot 001$ \\
\hline Black other ${ }^{\dagger}$ & $2 \cdot 1$ & $1 \cdot 61$ & $(1 \cdot 48-1 \cdot 76)$ & $<0 \cdot 001$ & $1 \cdot 7$ & 1.64 & $(1 \cdot 21-2 \cdot 21)$ & $<0.001$ & $0 \cdot 7$ & 0.64 & $(0.57-0.70)$ & $<0 \cdot 001$ & $1 \cdot 3$ & $0 \cdot 36$ & $(0 \cdot 32-0 \cdot 39)$ & $<0 \cdot 001$ \\
\hline Other ethnicity & $2 \cdot 6$ & $1 \cdot 19$ & $(1 \cdot 10-1 \cdot 28)$ & $<0 \cdot 001$ & $3 \cdot 5$ & $1 \cdot 34$ & $(1 \cdot 09-1 \cdot 65)$ & $0 \cdot 010$ & $1 \cdot 3$ & $0 \cdot 65$ & $(0 \cdot 59-0 \cdot 72)$ & $<0 \cdot 001$ & $1 \cdot 4$ & $0 \cdot 60$ & $(0 \cdot 56-0.65)$ & $<0 \cdot 001$ \\
\hline \multicolumn{17}{|l|}{ IMD quintile } \\
\hline 1 (least deprived) & $7 \cdot 6$ & 1 & & & $7 \cdot 2$ & 1 & & & $15 \cdot 7$ & 1 & & & $14 \cdot 6$ & 1 & & \\
\hline 2 & $10 \cdot 2$ & $1 \cdot 06$ & $(1 \cdot 00-1 \cdot 12)$ & $0 \cdot 050$ & $9 \cdot 9$ & $1 \cdot 04$ & $(0 \cdot 87-1 \cdot 24)$ & $0 \cdot 700$ & $17 \cdot 3$ & 0.99 & $(0 \cdot 95-1 \cdot 03)$ & $0 \cdot 480$ & $16 \cdot 5$ & $0 \cdot 98$ & $(0 \cdot 96-1 \cdot 01)$ & $0 \cdot 190$ \\
\hline 3 & $15 \cdot 7$ & $\mathbf{1 \cdot 2 0}$ & $(1 \cdot 14-1 \cdot 26)$ & $<0 \cdot 001$ & $17 \cdot 0$ & $1 \cdot 17$ & $(1 \cdot 00-1 \cdot 38)$ & $0 \cdot 060$ & $19 \cdot 1$ & 0.98 & $(0 \cdot 94-1 \cdot 02)$ & $0 \cdot 270$ & $19 \cdot 4$ & 0.94 & $(0.92-0.96)$ & $<0 \cdot 001$ \\
\hline 4 & $30 \cdot 1$ & $1 \cdot 48$ & $(1 \cdot 40-1 \cdot 55)$ & $<0 \cdot 001$ & $32 \cdot 4$ & $1 \cdot 28$ & $(1 \cdot 10-1 \cdot 49)$ & $<0 \cdot 001$ & $23 \cdot 6$ & 0.92 & $(0 \cdot 88-0.95)$ & $<0 \cdot 001$ & $23 \cdot 7$ & 0.92 & $(0 \cdot 90-0.95)$ & $<0 \cdot 001$ \\
\hline 5 (most deprived) & $36 \cdot 4$ & $1 \cdot 67$ & $(1 \cdot 59-1 \cdot 75)$ & $<0 \cdot 001$ & $33 \cdot 6$ & $1 \cdot 36$ & $(1 \cdot 17-1 \cdot 59)$ & $<0 \cdot 001$ & $24 \cdot 3$ & 0.92 & $(0 \cdot 88-0 \cdot 95)$ & $<0 \cdot 001$ & $25 \cdot 8$ & $0 \cdot 89$ & $(0 \cdot 87-0.92)$ & $<0 \cdot 001$ \\
\hline \multicolumn{17}{|l|}{ Sexual orientation } \\
\hline Heterosexual men & $25 \cdot 8$ & 1 & & & $15 \cdot 3$ & 1 & & & $50 \cdot 3$ & 1 & & & $32 \cdot 9$ & 1 & & \\
\hline MSM & $47 \cdot 6$ & $5 \cdot 90$ & $(5 \cdot 72-6 \cdot 09)$ & $<0 \cdot 001$ & $75 \cdot 9$ & $11 \cdot 74$ & $(10 \cdot 55-13 \cdot 06)$ & $<0.001$ & $4 \cdot 4$ & $0 \cdot 34$ & $(0 \cdot 32-0 \cdot 36)$ & $<0 \cdot 001$ & $4 \cdot 2$ & $\mathbf{0} \cdot 27$ & $(0 \cdot 26-0 \cdot 28)$ & $<0 \cdot 001$ \\
\hline Women & $26 \cdot 7$ & $0 \cdot 57$ & $(0 \cdot 55-0.59)$ & $<0.001$ & $8 \cdot 7$ & $0 \cdot 36$ & $(0 \cdot 31-0 \cdot 42)$ & $<0.001$ & $45 \cdot 3$ & $1 \cdot 19$ & $(1 \cdot 16-1 \cdot 22)$ & $<0 \cdot 001$ & $62 \cdot 9$ & 0.51 & $(0 \cdot 50-0 \cdot 51)$ & $<0 \cdot 001$ \\
\hline \multicolumn{17}{|l|}{ Age group, years } \\
\hline $15-24$ & $39 \cdot 3$ & 1 & & & $13 \cdot 2$ & 1 & & & $40 \cdot 6$ & 1 & & & $52 \cdot 7$ & 1 & & \\
\hline $25-34$ & $36 \cdot 0$ & $0 \cdot 72$ & $(0 \cdot 70-0 \cdot 74)$ & $<0 \cdot 001$ & 33.6 & 1.65 & $(1 \cdot 46-1 \cdot 87)$ & $<0.001$ & $33 \cdot 1$ & $1 \cdot 06$ & $(1 \cdot 03-1 \cdot 09)$ & $<0 \cdot 001$ & $30 \cdot 3$ & 0.68 & $(0.67-0.69)$ & $<0 \cdot 001$ \\
\hline $35-44$ & $15 \cdot 7$ & 0.55 & $(0.53-0.57)$ & $<0 \cdot 001$ & $28 \cdot 3$ & $2 \cdot 24$ & $(1 \cdot 98-2 \cdot 54)$ & $<0.001$ & $14 \cdot 0$ & $1 \cdot 12$ & $(1 \cdot 08-1 \cdot 16)$ & $<0.001$ & $9 \cdot 8$ & 0.54 & $(0.53-0.56)$ & $<0 \cdot 001$ \\
\hline $45-64$ & $8 \cdot 5$ & $0 \cdot 38$ & $(0 \cdot 36-0 \cdot 40)$ & $<0 \cdot 001$ & $23 \cdot 5$ & $2 \cdot 31$ & $(2 \cdot 03-2 \cdot 63)$ & $<0.001$ & $11 \cdot 4$ & $1 \cdot 29$ & $(1 \cdot 24-1 \cdot 34)$ & $<0 \cdot 001$ & $6 \cdot 6$ & $0 \cdot 48$ & $(0 \cdot 46-0 \cdot 50)$ & $<0 \cdot 001$ \\
\hline$\geqslant 65$ & $0 \cdot 4$ & $0 \cdot 21$ & $(0 \cdot 17-0 \cdot 25)$ & $<0.001$ & $1 \cdot 5$ & $1 \cdot 72$ & $(1 \cdot 26-2 \cdot 36)$ & $<0.001$ & $0 \cdot 8$ & $1 \cdot 09$ & $(0 \cdot 95-1 \cdot 23)$ & $0 \cdot 210$ & 0.6 & $0 \cdot 41$ & $(0 \cdot 37-0 \cdot 46)$ & $<0 \cdot 001$ \\
\hline
\end{tabular}

CI, Confidence interval, IMD, index of multiple deprivation; MSM, men who have sex with men.

* Syphilis is defined as primary, secondary and early latent syphilis

$\dagger$ Black other: non-Caribbean/non-African black ethnicity 
observations from all STI services throughout the country and over 450000 diagnoses of STIs reported in 2013. This enabled derivation of robust populationbased estimates of the diagnosis rates of common STIs both at a national and local level [22].

Evidence suggests that most STIs diagnosed in England are detected at a sexual health clinic or are referred to a sexual health clinic from general practice $[30,31]$.

The clear disparity in sexual ill-health by ethnic group, with those from black ethnic minorities having higher rates of specific STI diagnoses found in this study, is consistent with previous studies based in the UK, as well in United States [1, 5, 19]. In line with other studies, the results of this analysis confirm SED as a key determinant of poor health outcomes [32, 33].

SED only partly explains ethnic differences in STI diagnosis rates. It is likely that the high rates of STI diagnoses seen in black ethnic minorities relate to a complex interaction of structural determinants such as cultural, social and economic conditions and individual-level factors.

Structural determinants influence the health of communities as a whole and include education, employment, access to services and job security [34].

The individual-level factors include high-risk behaviours such as unsafe sexual practices [35], drug-injecting practices [34], and health-seeking behaviour, especially the use of treatment and screening services [36]. There is limited evidence in health-seeking behaviour by ethnicity; however, data from the second British National Survey of Sexual Attitudes and Lifestyles (Natsal 2000) show that the proportion of people of black Caribbean ethnicity reporting sexual health clinic attendance and STI diagnosis is higher compared to those of white ethnicity [4]. A higher prevalence of infections in black ethnic minorities may make them more likely to attend a sexual health clinic. However, other factors could influence the health-seeking behaviour.

It is well documented that an individual's sexual risk behaviour occurs within the context of a sexual partnership or partnerships within a wider sexual network and background prevalence of untreated disease [37]. These more proximal determinants of risk also occur within the context of broader social and structural determinants such as racial discrimination perception [38, 39]. In particular, perceived racial segregation acts directly upon the patterns of the sexual networks. The correlation between geographical proximity and a sexual network is a key component of STI prevalence due to high probability of choosing another sexual partner within the network [40].

Disparities between groups are by definition community-level differences: the community is here intended as physical vicinity (e.g. neighbourhoods) and commonality of purpose [38].

Reducing STI transmission and acquisition risk in specific ethnic groups requires recognition of these contributing factors. Developing approaches that challenge the underlying social-structural drivers of vulnerability and behaviour are needed. Clinic and community-based interventions could involve counselling and social peer networks to deliver behavioural skill-based interventions such as sexual negotiation and risk perception.

The ethnic disparity in STI diagnosis rates is partially explained by SED, but behavioural and other factors are likely to contribute. To investigate and adjust for other potential predictors of the STI diagnosis rates by ethnicity, behavioural data from the proposed enhancement of GUMCADv2 can be taken into account in a future study. This proposed enhancement is to collect details on high-risk sexual behaviour, including the use of recreational drugs in a sexualized context, and these data will contribute to our understanding of the ethnic disparities in sexual health. Further research into understanding the drivers and context of sexual risk-taking behaviours using geospatial information in order to highlight sexual networks is also warranted.

\section{ACKNOWLEDGEMENTS}

This research received no specific grant from any funding agency, commercial or not-for-profit sectors.

\section{DECLARATION OF INTEREST}

None

\section{REFERENCES}

1. Millett GA, et al. Comparisons of disparities and risks of HIV infection in black and other men who have sex with men in Canada, UK, and USA: a meta-analysis. Lancet 2012; 380: 341-348.

2. Woestenberg PJ, et al. Comparison of STI-related consultations among ethnic groups in the Netherlands: an epidemiologic study using electronic records from general practices. BMC Family Practice 2015; 16: 1-8.

3. The UK Collaborative Group for HIV and STI Surveillance. Sexually transmitted infections in black 
African and black Caribbean communities in the UK: 2008 report: Health Protection Agency, Centre for Infections; 2008.

4. Fenton KA, et al. Ethnic variations in sexual behaviour in Great Britain and risk of sexually transmitted infections: a probability survey. Lancet 2005; 365: 12461255.

5. Hughes G, et al. Comparison of risk factors for four sexually transmitted infections: results from a study of attenders at three genitourinary medicine clinics in England. Sexually Transmitted Infections 2000; 76: 262-267.

6. GOV.UK. Sexually transmitted infections (STIs): annual data tables (https://www.gov.uk/government/statistics/ sexually-transmitted-infections-stis-annual-data-tables). Accessed 16 March 2016.

7. Savage EJ, et al. Rapid increase in gonorrhoea and syphilis diagnoses in England in 2011. Eurosurveillance 2012; 17: 195-196.

8. Marmot M. Social determinants of health inequalities. Lancet 2005; 365: 1099-1104.

9. Shavers VL. Measurement of socioeconomic status in health disparities research. Journal of the National Medical Association 2007; 99: 1013-1023.

10. Butler DC, et al. Measures of social deprivation that predict health care access and need within a rational area of primary care service delivery. Health Services Research 2013; 48: 539-559.

11. Kershaw KN, Albrecht SS, Carnethon MR. Racial and ethnic residential segregation, the neighborhood socioeconomic environment, and obesity among blacks and Mexican Americans. American Journal of Epidemiology 2013; 177: 299-309.

12. White $\mathbf{K}$, et al. Racial/ethnic residential segregation and self-reported hypertension among US- and foreign-born blacks in New York City. American Journal of Hypertension 2011; 24: 904-910.

13. Krieger J, Higgins DL. Housing and health: time again for public health action. American Journal of Public Health 2002; 92: 758-768.

14. Newton JN, et al. Changes in health in England, with analysis by English regions and areas of deprivation, 1990-2013: a systematic analysis for the Global Burden of Disease Study 2013. Lancet 2015; 386: 2257-2274.

15. Moonesinghe R, et al. Linear and non-linear associations of gonorrhea diagnosis rates with social determinants of health. International Journal of Environmental Research and Public Health 2012; 9: 3149-3165.

16. Hughes GJ, Gorton R. Inequalities in the incidence of infectious disease in the North East of England: a population-based study. Epidemiology \& Infection 2015; 143: 189-201.

17. Pound P, Campbell R. Exploring the feasibility of theory synthesis: A worked example in the field of health related risk-taking. Social Science \& Medicine 2015; 124: $57-65$.

18. Gupta GR, et al. Structural approaches to HIV prevention. Lancet 2008; 372: 764-775.
19. Nazroo J, et al. The Black diaspora and health inequalities in the US and England: does where you go and how you get there make a difference? Sociology of Health \& Illness 2007; 29: 811-830.

20. Savage EJ, et al. Assessing the relationship between sexually transmitted infection rates, ethnic group and socio-economic deprivation in England. Sexually Transmitted Infections 2011; 87: A195-A196.

21. Office for National Statistics. Super Output Area (SOA) (webarchive.nationalarchives.gov.uk/20160105160709/ http://www.ons.gov.uk/ons/guide-method/geography/beginner-s-guide/census/super-output-areas--soas-/index. html). Accessed 16 March 2016.

22. Savage EJ, et al. Improving surveillance of sexually transmitted infections using mandatory electronic clinical reporting: the genitourinary medicine clinic activity dataset, England, 2009 to 2013. Eurosurveillance 2014; 19: 20981 .

23. GOV.UK. English indices of deprivation 2010 (https:// www.gov.uk/government/statistics/english-indices-of-deprivation-2010). Accessed 16 March 2016.

24. Payne R, Abel G. UK indices of multiple deprivation a way to make comparisons across constituent countries easier. Health Statistics Quarterly 2012; 53: 22-37.

25. Office for National Statistics. Population estimates (http:// www.ons.gov.uk/peoplepopulationandcommunity/populationandmigration/populationestimates). Accessed 16 March 2016.

26. StataCorp. Stata Statistical Software: Release 13. College Station, TX: StataCorp LP, 2013.

27. GOV.UK. Genitourinary medicine clinic activity dataset (GUMCADv3) pilot (https://www.gov.uk/guidance/ genitourinary-medicine-clinic-activity-dataset-gumcadv3pilot). Accessed 16 March 2016.

28. Public Health England. Sexually transmitted infections and chlamydia screening in England, 2014. Health Protection Report (https://www.gov.uk/government/ uploads/system/uploads/attachment_data/file/437433/hpr2215_STI_NCSP_v6.pdf). Accessed 16 March 2016.

29. GOV.UK. Chlamydia Testing Activity Dataset (CTAD): specification and technical guidance (https://www.gov.uk/ government/publications/chlamydia-testing-activity-dataset-ctad-specification-and-technical-guidance). Accessed 16 March 2016.

30. Hughes G, Field N. The epidemiology of sexually transmitted infections in the UK: impact of behavior, services and interventions. Future Microbiology 2015; 10: $35-51$.

31. Wetten S, et al. Diagnosis and treatment of chlamydia and gonorrhoea in general practice in England 2000 2011: a population-based study using data from the UK Clinical Practice Research Datalink. BMJ Open 2015; 5: e007776.

32. Zhang X, et al. The effects of deprivation and relative deprivation on self-reported morbidity in England: an area-level ecological study. International Journal of Health Geographics 2013; 12: 1-11.

33. Mazur J, et al. Subjective health of adolescents from families in receipt of social assistance. Public Health. 
34. Dean HD, Fenton KA. Addressing social determinants of health in the prevention and control of HIV/AIDS, viral hepatitis, sexually transmitted infections, and tuberculosis. Public Health Reports 2012; 372: 125-132.

35. Gerver SM, et al. Sexual risk behaviours and sexual health outcomes among heterosexual black Caribbeans: comparing sexually transmitted infection clinic attendees and national probability survey respondents. International Journal of STD \& AIDS 2011; 22: 85-90.

36. Gerressu M, et al. The importance of distinguishing between black Caribbeans and Africans in understanding sexual risk and care-seeking behaviours for sexually transmitted infections: evidence from a large survey of people attending genitourinary medicine clinics in England. Journal of Public Health 2012; 34: $411-420$.

37. Beyrer C, et al. Global epidemiology of HIV infection in men who have sex with men. Lancet 2012; 380: 367-377.

38. Hogben M, Leichliter JS. Social determinants and sexually transmitted disease disparities. Sexually Transmitted Diseases 2008; 35: S13-18.

39. Bowleg L, et al. Racial discrimination, social support, and sexual HIV risk among Black heterosexual men. AIDS and Behavior 2013; 17: 407-418.

40. Westercamp N, et al. Spatial distribution and cluster analysis of sexual risk behaviors reported by young men in Kisumu, Kenya. International Journal of Health Geographics 2010; 9: 24. 